\title{
Himenópteros parasitóides associados a pupas de Methona themisto (Lepidoptera, Nymphalidae) em Florianópolis, Santa Catarina, Brasil
}

\author{
Cristiane Nardi ${ }^{1}$, Tânia M. Guerra ${ }^{2}$, Afonso I. Orth ${ }^{3} \&$ Marcelo T. Tavares ${ }^{4}$ \\ 1. Laboratório de Biologia de Insetos, Escola Superior de Agricultura Luiz de Queiroz (ESALQ/USP), Caixa Postal 11, $13418-900$ \\ Piracicaba, SP. (cnardi@esalq.usp.br). \\ 2. Colégio de Aplicação, Universidade Federal de Santa Catarina, Caixa Postal 476, 88040-900 Florianópolis, SC. \\ 3. Laboratório de Entomologia Agrícola, Universidade Federal de Santa Catarina. \\ 4. Laboratório de Biodiversidade de Insetos, Departamento de Ciências Biológicas, Universidade Federal do Espírito Santo, Av. Marechal \\ Campos, 1468, 29040-090 Vitória, ES.
}

\begin{abstract}
Hymenopterous parasitoids associated to pupae of Methona themisto (Lepidoptera, Nymphalidae) in Florianópolis, Santa Catarina, Brazil. The goals of this study were to know the parasitoid guild associated to pupae of Methona themisto (Hübner, 1818), evaluate the relative abundance of each species of parasitoid and the effect of parasitism on the host mortality. A total of 160 pupae of $M$. themisto were collected, 80 in October 2001 and 80 in October 2002. They were maintained at $25 \pm 2,0^{\circ} \mathrm{C}$ and photophase of 14 hours, daily observed until parasitoid or butterfly emergence. Five parasitoid species were obtained from $M$. themisto pupae: Brachymeria annulata (Fabricius, 1793), B. mnestor (Walker, 1841) (Chalcididae), Neotheronia sp. (Ichneumonidae), Palmistichus elaeisis Delvare \& LaSalle, 1993, and Tetrastichus sp. (Eulophidae). Only the genera Brachymeria and Neotheronia occurred in both years of the study, the former was more abundant in 2001 and the latter in 2002. The other species of parasitoids occurred only in the second year of the study. These are the first records of B. annulata, B. mnestor, Neotheronia sp. and P. elaeisis as parasitoids of $M$. themisto.
\end{abstract}

KEYWORDS. Ithomiinae, parasitism, parasitoid guild.

RESUMO. Os objetivos deste estudo foram conhecer a guilda de parasitóides associados a pupas de Methona themisto (Hübner, 1818), avaliando a abundância relativa de cada espécie de parasitóide e o efeito do parasitoidismo na mortalidade do hospedeiro. Foram coletadas 160 pupas de $M$. themisto, 80 em outubro de 2001 e 80 em outubro de 2002 . Essas foram mantidas em temperatura de $25 \pm 2,0^{\circ} \mathrm{C}$ e fotofase de 14 horas, sendo diariamente observadas, até a emergência de parasitóides ou borboletas. Os seguintes parasitóides foram obtidos das pupas de M. themisto: Brachymeria annulata (Fabricius, 1793), B. mnestor (Walker, 1841) (Chalcididae), Neotheronia sp. (Ichneumonidae), Palmistichus elaeisis Delvare \& LaSalle, 1996 e Tetrastichus sp. (Eulophidae). Somente os gêneros Brachymeria e Neotheronia ocorreram nos dois anos de estudo, sendo que o primeiro foi mais abundante em 2001 e o último, em 2002. As outras espécies de parasitóides ocorreram somente no segundo ano de estudo. Este é o primeiro relato da associação de B. annulata, B. mnestor, Neotheronia sp. e P. elaeisis com M. themisto.

PALAVRAS-CHAVE. Ithomiinae, parasitoidismo, guilda de parasitóides.

Nos ecossistemas, o parasitoidismo é o responsável por grande parte da regulação dos níveis populacionais de insetos, sendo que um herbívoro pode ser atacado por várias espécies de parasitóides (MAY, 1988). Sendo assim, a caracterização dos hospedeiros e de seus parasitóides é uma estratégia importante para a elucidação dos aspectos biológicos e ecológicos destes organismos.

Methona themisto (Hübner, 1818) é comum nas regiões Sul e Sudeste do Brasil, ocorrendo desde o estado do Pará até o Rio Grande do Sul (Silva et al., 1968), principalmente em áreas urbanizadas (RUSZCZYK, 1986; RODRIGUEs et al., 1993). Suas lagartas alimentam-se exclusivamente de folhas de Brunfelsia Linnaeus, 1822 (Solanaceae), sendo geralmente associadas ao manacá, Brunfelsia uniflora (Pohl) D. Don, arbusto ornamental nativo do Brasil. Em épocas em que a população aumenta, as larvas de $M$. themisto podem ocasionar o desfolhamento total das plantas (SiLva et al., 1968; RUSZCZYK \& NASCIMENTO, 1999).

Apesar de sua ampla distribuição no Brasil e alta tolerância aos distúrbios do ambiente urbano, $M$. themisto tem sido pouco estudada e muitos aspectos de sua bioecologia ainda são desconhecidos. Sobre os inimigos naturais dessa espécie, há relatos do taquinídeo Zigofrontina sp. (Diptera) como parasitóide de larvas (FIGUEIREDO JúNIOR, 1939).

Neste estudo, objetivou-se conhecer a guilda de parasitóides associada a pupas de $M$. themisto obtidas em $B$. uniflora, identificando cada espécie e avaliando sua abundância relativa.

\section{MATERIAL E MÉTODOS}

Foram coletadas 160 pupas de $M$. themisto, oriundas de plantas adultas de B. uniflora, situadas no bairro Itacorubi $\left(27^{\circ} 35^{\prime} \mathrm{S}, 48^{\circ} 30^{\prime} \mathrm{W}\right)$, área urbana do município de Florianópolis, SC. Dessas pupas, 80 foram coletadas em outubro de 2001 e 80 em outubro de 2002.

As pupas foram individualizadas em tubos plásticos (4,0 cm de diâmetro e 7,5 cm de altura) com abertura forrada com tecido de náilon e mantidas em temperatura de $25 \pm$ $2,0^{\circ} \mathrm{C}$ e fotofase de 14 horas. Foram realizadas observações diárias até a emergência dos parasitóides e das borboletas (60 dias), que foram montados para identificação posterior. 
Estas pupas foram separadas em três categorias: parasitadas, que originaram parasitóides adultos; nãoparasitadas, que originaram borboletas; e mortas, que não originaram borboletas ou parasitóides.

Para comparar o número de parasitóides obtidos nos dois anos de estudo e o número de pupas de $M$. themisto mortas, parasitadas e não-parasitadas, foi realizado o teste do $\chi^{2}(\alpha=0,05)$. Para essas comparações, as duas espécies de Brachymeria foram analisadas conjuntamente, pois para avaliações de abundância, a identificação foi realizada apenas para gênero.

O material-testemunho encontra-se depositado na Coleção Entomológica do Departamento de Ciências Biológicas da Universidade Federal do Espírito Santo (UFES) e do Centro de Ciências Agrárias da Universidade Federal de Santa Catarina. A identificação dos parasitóides foi realizada baseando-se em BouCEK (1992) e Boucek \& Delvare (1992), para os calcidídeos; SchaufF et al. (1997) e Delvare \& LaSalle (1993), para os eulofídeos; e Townes (1969), para os ichneumonídeos.

\section{RESULTADOS E DISCUSSÃO}

Foram obtidos os seguintes parasitóides associados a pupas de $M$. themisto: Brachymeria annulata (Fabricius, 1793); B. mnestor (Walker, 1841) (Chalcididae); Neotheronia sp. (Ichneumonidae); Palmistichus elaeisis Delvare \& LaSalle, 1993 e Tetrastichus sp. (Eulophidae). A identificação das espécies de Neotheronia e Tetrastichus não foi possível, uma vez que esses gêneros incluem muitas espécies neotropicais e, dentre as descrições existentes para a maioria das espécies, nenhuma permitiu a caracterização e diferenciação dos indivíduos coletados.

BouceK (1992) considerou B. annulata e B. mnestor como endoparasitóides solitários de pupas ou pré-pupas de Lepidoptera, as quais podem também ocorrer como hiperparasitóides de Diptera e Ichneumonoidea. No presente estudo, não foi possível confirmar se essas espécies atuaram como parasitóides primários ou hiperparasitóides, mas ambas apresentaram hábito solitário. Brachymeria annulata foi encontrada nos dois anos de estudo, enquanto que B. mnestor apenas no segundo ano. Esses são os primeiros registros de associação dessas espécies de Brachymeria com $M$. themisto.

A espécie de Neotheronia desenvolveu-se como endoparasitóide solitário de $M$. themisto. Segundo GAULD (1988), as espécies de Neotheronia são ecto- ou endoparasitóides idiobiontes de pupas de Lepidoptera. Townes (1969) cita que estas espécies podem se desenvolver como hiperparasitóides de Ichneumonoidea. Neste trabalho não foi possível confirmar sua atuação como parasitóide primário ou hiperparasitóide. No entanto, este é o primeiro relato de associação de Neotheronia $\operatorname{com} M$. themisto.

Palmistichus elaeisis tem sido associado a pupas de Cotesia sp. (Braconidae), posturas de Hispoleptis subfasciata Pic, 1938 (Coleoptera, Chrysomelidae, Hispinae) e às espécies de lepidópteros Eupseudosoma involuta (Sepp, 1852) (Arctiidae) e Euselasia eucerus Hewitson, 1872 (Riodinidae) (Delvare \& Lasalle, 1993).
Bittencourt \& Berti Filho (1999, 2004) também constataram $P$. elaeisis associada a várias espécies de Lepidoptera, pertencentes a Crambidae, Noctuidae e Geometridae. Neste estudo foi constatada a associação de $P$. elaeisis com $M$. themisto pela primeira vez, sendo esse parasitóide obtido de duas pupas, numa das quais emergiu juntamente com exemplares de Tetrastichus sp.

As espécies de Tetrastichus atuam como parasitóides primários de larvas e pupas de Diptera e Lepidoptera e como hiperparasitóides de imaturos de Hymenoptera (Noyes, 2002). Na Argentina, Tetrastichus sp. foi constatada como parasitóide de pupas de $M$. confusa psamathe Godman \& Salvin, 1898 sobre Brunfelsia australis Benth. (RomÁn, 1996). Entretanto, no Brasil, não havia sido registrada, até o momento, a associação deste parasitóide com espécies de Methona.

Neotheronia e as duas espécies de Brachymeria ocorreram nos dois anos de estudo, sendo que o número de indivíduos obtidos para cada gênero foi significativamente diferente entre os períodos $\left(\chi^{2}=38,48\right.$; $\mathrm{P}<0,05)$. Com relação à abundância e freqüência dos parasitóides, verificou-se que, no primeiro ano, Neotheronia sp. foi mais freqüente, enquanto que, no segundo ano, o parasitoidismo pelas espécies de Brachymeria foi superior (Tab. I). Dentre os fatores que podem ter influenciado a variação na abundância dessas espécies de parasitóides, pode-se citar os abióticos, como temperatura e umidade requeridas pelos indivíduos de cada espécie, e os bióticos, como a disponibilidade de hospedeiros, a ação de inimigos naturais sobre esses parasitóides, a competição intraespecífica e a densidade populacional (HufFAKER et al., 1984). Além disso, de acordo com VAN Den Bosch et al. (1985) e Godfray (1994), a competição interespecífica é um aspecto determinante da abundância de parasitóides de uma guilda, ocorrendo uma ação recíproca entre a densidade numérica das populações, de modo que, no período em que a densidade de uma delas aumenta, a da outra diminui.

Tetrastichus sp. e P. elaeisis ocorreram somente no segundo ano de estudo. De uma das pupas que originaram eulofídeos, emergiram 96 exemplares de $P$. elaeisis e, de outra, emergiram 29 indivíduos de Tetrastichus sp. e dois de P. elaeisis (Tab. I). A obtenção de Tetrastichus sp. e P. elaiesis de única pupa pode estar relacionada à estratégia hiperparasitóide de uma dessas espécies ou a um caso de parasitoidismo múltiplo. No entanto, nesse trabalho não foi possível determinar se essas espécies ocorreram como parasitóides primários de $M$. themisto, ou se uma das espécies de parasitóide ocorreu como hospedeira da outra.

O número de pupas de $M$. themisto parasitadas, não parasitadas e mortas apresentou-se diferente de um ano para outro $\left(\chi^{2}=37,09 ; \mathrm{p}<0,05\right)$ (Tab. II). Dentre as pupas coletadas, as não-parasitadas foram mais abundantes no primeiro ano, enquanto o parasitoidismo (pupas parasitadas) foi maior no segundo ano. Esse fato pode ser explicado baseando-se em LASALLE \& GAULD (1991), GodFray (1994) e Hastings \& GodFray (1999), que afirmam que os parasitóides podem reagir numericamente à densidade de hospedeiros disponíveis, concentrandose gradualmente onde esses são mais abundantes. Assim, um ciclo com baixo controle dos parasitóides sobre a 
Tabela I. Abundância e freqüência dos parasitóides de pupas Methona themisto nos anos de 2001 e 2002, em Florianópolis, SC. Letras diferentes na mesma linha referem-se a resultados significativamente diferentes entre si $(\alpha=0,05)$.

\begin{tabular}{|c|c|c|c|c|c|}
\hline \multicolumn{2}{|c|}{ Parasitóides } & \multicolumn{2}{|c|}{2001} & \multicolumn{2}{|c|}{2002} \\
\hline Família & Espécie & Abundância & Freqüência (\%) & Abundância & Freqüência (\%) \\
\hline Chalcididae & Brachymeria spp. & $2^{b}$ & 16,7 & $42^{a}$ & 93,33 \\
\hline Ichneumonidae & Neotheronia sp. & $10^{\mathrm{a}}$ & 83,3 & $1^{b}$ & 2,22 \\
\hline \multirow[t]{3}{*}{ Eulophidae } & Tetrastichus sp. & - & - & 2 & 4,45 \\
\hline & Palmistichus elaeisis & - & - & & \\
\hline & Total & 12 & 100 & 45 & 100 \\
\hline
\end{tabular}

Tabela II. Número de pupas de Methona themisto parasitadas, nãoparasitadas e mortas, obtidas em 2001 e 2002, em Florianópolis, SC. Letras diferentes na mesma linha referem-se a resultados significativamente diferentes entre si $(\alpha=0,05)$.

\begin{tabular}{lcc}
\hline Categorias & 2001 & 2002 \\
& Abundância & Frequência Abundância Frequência
\end{tabular}

\begin{tabular}{llcrc} 
& \multicolumn{5}{c}{$(\%)$} & $(\%)$ \\
\hline Parasitadas & $12^{\mathrm{b}}$ & 15,0 & $48^{\mathrm{a}}$ & 60,0 \\
Não-parasitadas & $39^{\mathrm{a}}$ & 48,8 & $24^{\mathrm{b}}$ & 30,0 \\
Mortas & $29^{\mathrm{a}}$ & 36,2 & $8^{\mathrm{b}}$ & 10,0 \\
\hline Total & 80 & 100,0 & 80 & 100,0 \\
\hline
\end{tabular}

população da espécie hospedeira possibilitaria um incremento na abundância desta. Havendo maior disponibilidade destes, o aumento na densidade numérica dos inimigos naturais seria favorecido no ciclo seguinte. Embora se tenha verificado um incremento no número de pupas de $M$. themisto parasitadas, deve-se considerar que estas taxas de parasitoidismo são resultantes de fatores como densidade do hospedeiro no campo e competição entre as populações de inimigos naturais, que não foram avaliados nesse estudo. Sendo assim, sugere-se que novos estudos sejam realizados com o objetivo de relacionar a variação dos níveis populacionais de $M$. themisto com a freqüência de inimigos naturais em campo.

Agradecimentos. Ao Dr. Alex Pires de Oliveira Nuñer (Engenharia de Aqüicultura/UFSC), pelas sugestões no manuscrito; aos revisores, em especial ao Dr. Celso de Oliveira Azevedo, pela análise criteriosa e sugestões enriquecedoras; ao $\mathrm{CNPq}$, pela bolsa de recém-doutor concedida a T. M. Guerra.

\section{REFERÊNCIAS BIBLIOGRÁFICAS}

Bittencourt, M. A. L. \& Berti Filho, E. 1999. Preferência de Palmistichus elaeisis por pupas de diferentes lepidópteros praga. Scientia Agricola 56(4):1281-1283.

2004. Thermal requirements for the development of Palmistichus elaeisis (Hymenoptera, Eulophidae) in pupae of five lepidopteran species. Iheringia, Série Zoologia. 94(3):321-323.

Boucek, Z. 1992. The New World genera of Chalcididae. Memoirs of the American Entomological Institute 53:49-118; 443-460.

Boucek, Z. \& Delvare, G. 1992. The identities of species described or classified under Chalcis by J.C. Fabricius. Memoirs of the
American Entomological Institute 53:11-48.

Delvare, G. \& LaSalle, J. 1993. A new genus of Tetrastichinae (Hymenoptera: Eulophidae) from the Neotropical Region, with the description of a new species parasitic on key pests of oil palm. Journal of Natural History 27:435-444.

FigueIREDo JÚNIOR, E. R. 1939. Notas sobre a Thridia themisto Huebn., 1823, praga do manacá. Boletim Biológico 4(3):512-515.

GAULD, I. D. 1988. Evolutionary patterns of host utilization by ichneumonoid parasitoids (Hymenoptera: Ichneumonidae and Braconidae). Biological Journal of Linnaeus Society 35:351-377.

Godfray, H. C. J. 1994. Parasitoids: behavioral and evolutionary ecology. New Jersey, Princeton University. $463 \mathrm{p}$.

Hastings, A. \& Godfray, H. C. J. 1999. Learning, host fidelity, and stability of host-parasitoid communities. American Naturalist 153(3):295-301.

Huffaker, C. B.; Berryman, A. A. \& Laing, J. E. 1984. Natural control of insect population. In: Huffaker, C. B. \& RabB, R. L. eds. Ecological Entomology. New York, John Wiley \& Sons. p.399-426.

LaSalle, J. \& Gauld, I. D. 1991. Parasitic Hymenoptera and the biodiversity crisis. Redia 74:315-334.

MAY, R. M. 1988. How many species are there on Earth? Science 241:1441-1449.

Noyes, J. S. 2002. Interactive Catalogue of World Chalcidoidea, 2001. Vancouver, Taxapad. CD-ROM.

Rodrigues, J. J. L.; Brown, K. S. JR. \& RuszczyK, A. 1993. Resources and conservation of Neotropical butterfly in urban forest fragments. Biological Conservation 64(1):3-9.

RomÁn, L. E. N. 1996. Morfologia de los estados inmaturos y aspectos biológicos de Tetrastichus sp. (Hymenoptera: Tetrastichidae), parasitóide de Methona confusa psamathe Godm. et Salv. (Lepidoptera: Ithomiidae). Neotrópica 42(107108):41-46.

RuszczyK, A. 1986. Ecologia urbana de borboletas, I. O gradiente de urbanização e a fauna de Porto Alegre, RS. Revista Brasileira de Biologia 46:255-264.

RuszczyK, A. \& Nascimento, E. S. 1999. Biologia dos adultos de Methona themisto (Hübner, 1818) (Lepidoptera, Nymphalidae, Ithomiinae) em praças públicas de Uberlândia, Minas Gerais, Brasil. Revista Brasileira de Biologia 59:577-583.

Schauff, M. E.; LaSalle, J. \& Coote, L. D. 1997. Eulophidae. In: Gibson, G. A. P.; Huber, J. T. \& Wooley, J. B. eds. Annotated keys to the genera of Nearctic Chalcidoidea (Hymenoptera). Ottawa, NRC Research. p.327-429.

Silva, A. G. D.; Gonçalves, C. R.; Galvão, D. M.; Gonçalves, A. J. L.; Gomes, J.; Silva, M. N. \& Simoni, L. 1968. Quarto catálogo dos insetos que vivem nas plantas do Brasil: seus parasitos e predadores. Rio de Janeiro, Ministério da Agricultura. Parte II, $1^{\circ}$ Tomo. 622p.

Townes, H. 1969. The genera of Ichneumonidae (Part 1). Memoirs of the American Entomological Institute 11:1-300.

Van Den Bosch, R.; Messenger, P. S. \& Gutierrez, A. P. 1985. An introduction to biological control. New York, Plenum. 247p.

Recebido em março de 2004. Re-submetido em novembro de 2005. Aceito em junho de 2006. ISSN 0073-4721

Artigo disponível em: www.scielo.br/isz

Iheringia, Sér. Zool., Porto Alegre, 96(3):373-375, 30 de setembro de 2006 